\title{
Microscopic Mechanism on Giant Photoeffect in Proton Transport Through Graphene Membranes
}

\author{
Liming Guan ${ }^{1, \dagger}$, Beidou Guo ${ }^{1,2, \dagger}$, Xinrui Jia ${ }^{1,2}$, Guancai Xie ${ }^{1,2}$, Jian Ru Gong 1,2,* \\ ${ }^{1}$ Chinese Academy of Sciences (CAS) Center for Excellence in Nanoscience, CAS Key Laboratory of Nanosystem and Hierarchy \\ Fabrication, National Center for Nanoscience and Technology, Beijing 100190, China. \\ ${ }^{2}$ University of Chinese Academy of Sciences, Beijing 100049, China.
}

\begin{abstract}
Graphene monolayers are permeable to thermal protons and impermeable to other atoms and molecules, exhibiting their potential applications in fuel cell technologies and hydrogen isotope separation. Furthermore, the giant photoeffect in proton transport through catalytically activated graphene membranes was reported by Geim et al. Their experiment showed that the synergy between illumination and the catalytically active metal plays a key role in this photoeffect. Geim et al.
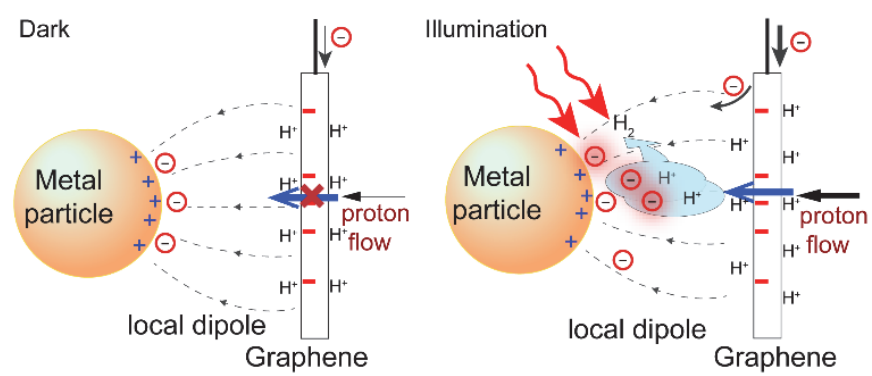
suggested that the local photovoltage created between metal nanoparticles and graphene could funnel protons and electrons toward the metal nanoparticles for the production of hydrogen, while repelling holes away from them, causing the giant photoeffect. However, based on static electric field theory, this explanation is not convincing and the work lacks an analysis on the microscopic mechanism of this effect. Herein, we provide the exact microscopic mechanism behind this phenomenon. In semi-metal pristine graphene, most photon excited hot electrons relax to lower energy states within a timescale of $10^{-12} \mathrm{~s}$, while the typical timescale of a chemical reaction is $10^{-6} \mathrm{~s}$. Thus, hot electrons excited by incident photons relax to lower energy states before reacting with protons through the graphene. When graphene is decorated with metal, electron transfer between the graphene and the metal, induced by different work functions, would result in the formation of interface dipoles. When using metals such as $\mathrm{Pt}, \mathrm{Pd}, \mathrm{Ni}$, etc., which can strongly interact with graphene, local dipoles form. Protons are trapped around the negative poles of the local dipoles, while electrons are around the positive poles. Upon illumination, the electrons are excited to metastable excited states with higher energy levels. Due to the energy barriers around them, the free electrons in the metastable excited states will have a relatively longer lifetime, which facilitates the production of hydrogen through their effective reaction with protons that permeated through the graphene. The concentration of high-energy electrons under illumination was estimated, and the results showed that more electrons are energized to the excited state with strong illumination. According to the analysis, the giant photoeffect in proton transport through the catalytically activated graphene membrane is attributed to long-lived hot electrons and a fast proton transport rate. Since there is no change in the activation energy of the reaction, the metal catalyst increases the rate of the reaction by increasing the number of successful collisions between the reactants to produce the significant photoeffect. This might lead to a new microscopic mechanism that clarifies the role of the catalyst in improving the efficiency of photo(electro)catalytic reactions.
\end{abstract}

Key Words: Graphene; Proton transport; Dipole; Hot electron; Hydrogen

Received: July 25, 2020; Revised: September 4, 2020; Accepted: September 7, 2020; Published online: September 11, 2020.

${ }^{*}$ Corresponding author. Email: gongjr@nanoctr.cn; Tel.: +86-10-82545649.

†These authors contributed equally to this work.

The project was supported by the Strategic Priority Research Program of CAS (XDB36030000), the National Natural Science Foundation of China (21422303, 21573049, 21872043), the National Basic Research Plan, China (2016YFA0201600), the Beijing Natural Science Foundation, China (2142036), the Youth Innovation Promotion Association and the Special Program of "One Belt One Road" of CAS.

中科院战略重点研究项目(XDB36030000), 国家自然科学基金项目(21422303, 21573049, 21872043), 国家重点研发项目(2016YFA0201600), 北京自然 科学基金项目(2142036), 中国科学院青年创新促进会及中国科学院 “一带一路” 专项资助

(C) Editorial office of Acta Physico-Chimica Sinica 


\title{
石墨烯膜质子传输巨大光效应的微观机理
}

\author{
关黎明 $1, \dagger$ ，郭北斗 $1,2, \uparrow ，$ 贾金荃芯 1,2 , 谢关才 1,2 , 宫建茹 $1,2,{ }^{*}$ \\ 1 国家纳米科学中心, 中国科学院纳米系统与多级次重点实验室, 中国科学院纳米科学卓越创新中心, 北京 100190 \\ 2 中国科学院大学, 北京 100049
}

\begin{abstract}
摘要: 单层石墨烯已被证明对质子是可渗透的, 而对其它原子和分子不可渗透, 这一特性在燃料电池和氢同位素分离等 方面具有潜在的应用。Geim等人报道了催化活化石墨烯膜质子传输的巨大光效应。其实验表明, 光照和具有催化活性金 属纳米颗粒的协同作用在这种光效应中起关键作用。Geim等人认为巨大光效应是由金属纳米颗粒和石墨烯之间产生的 局部光电压引起的。局部光电压将质子和电子传送至金属纳米颗粒以产生氢气，同时将空穴排斥使之远离。但是，根据 静电场理论, 这种解释并不能令人信服, 并且在他们的工作中也没有此效应的微观机理分析。我们在此文中提出了一种 该现象背后的确切微观机制。对于具有半金属性质的石墨烯, 光激发的大多数热电子会在皮秒时间内驰豫到较低的能态, 而发生化学反应所需的时间一般为纳秒范围。因此, 在单一石墨烯的情况下, 入射光激发的热电子在与透过石墨烯的质 子反应之前就已驰豫到较低的能态。当用金属粒子修饰石墨烯时, 由功函数不同引起的电子转移会导致界面偶极子的形 成。当金属为可与石墨烯具有相互强烈作用的 Pt、 $\mathrm{Pd} 、 \mathrm{Ni}$ 等时, 就会形成局部偶极子。质子将被俘获在局部偶极子的负 极周围, 而电子则被俘获在正极附近。在光照射后, 被俘获的电子会被激发到具有更高能级的亚稳激发态。处于高活化 能的亚稳激发态的自由电子具有更长的寿命, 使得它有更充分的时间与透过石墨烯的质子发生化学反应。对光照情况下 高能电子的浓度的计算结果显示, 光照越强时被激发到激发态的电子越多。根据本文的分析, 质子通过催化活化石墨烯 膜的巨大光效应归因于较长寿命的热载流子和快速的质子传输速率。因为这一反应的活化能没有变化, 所以金属催化剂 是通过增加反应物之间成功碰撞的次数来增大反应速率, 从而产生显著的光效应。该工作可能揭示了催化剂在提高光(电) 催化反应效率方面的一种新微观机制。
\end{abstract}

关键词: 石墨烯; 质子传输; 偶极; 热电子; 氢气 中图分类号: 0649

\section{Introduction}

Graphene monolayers have been shown to be permeable to thermal protons, while impermeable to other atoms and molecules, displaying their potential applications such as fuel cell technologies and hydrogen isotope separation ${ }^{1-4}$. In theory, first-principle calculations have shown that the energy barrier for proton permeation is in the range of $1.17-3.0 \mathrm{eV}$ depending on the adsorption states of proton ${ }^{5-7}$. As for the experiment, it was found that proton permeation through graphene can be thermally activated with an energy barrier of $0.8 \mathrm{eV}{ }^{2}$. Further measurements using the hydrogen isotope deuterium demonstrated that this barrier is actually $\sim 1 \mathrm{eV}$ due to the increased energy of incoming protons by quantum oscillation ${ }^{3}$. These discrepancies between the barriers found in theory and those in experiments triggered debates about the exact microscopic mechanism behind the proton permeation through graphene. Various mechanisms, such as hydrogenation of graphene, quantum tunneling of thermal protons, have been used to explain such proton permeation ${ }^{8-10}$. Furthermore, Geim et al. ${ }^{11}$ reported the giant photoeffect in proton transport through catalytically-activated graphene. In their experiment, protons transport from a proton source of the $\mathrm{PdH}_{x}$ electrode through a monolayer graphene under a bias voltage, then react with electrons on the other side of graphene to generate hydrogen when catalytically-active metal nanoparticles were decorated on graphene in the dark. Under illumination, a strongly enhanced proton current as well as hydrogen flux can be observed, and the Faradaic efficiency is $100 \%$ at all proton current densities and the activation energy does not change noticeably. In contrast, if graphene is not effectively catalytically-activated, there is no enhancement of the proton current under illumination. Obviously, a synergy of illumination and the catalytic-active metal plays a key role in this photoeffect.

On the basis of the facts in the experiment, a few possible mechanisms were first ruled out for explaining the photoeffect ${ }^{11}$. They include (1) plasmonic effect, due to the lack of response in the presence of plasmon-active Au nanoparticles ${ }^{12,13}$, (2) photoinduced electrolysis of water, since the applied voltage is well below the thermodynamic voltage for water splitting and graphene is impermeable to hydrogen ${ }^{11,14,15}$, and (3) photoinduced hydrogenation of graphene, because the expected reduction in the energy barrier, that is, the activation energy, is not observed in the experiment ${ }^{9,11}$. Then, Geim et al. ${ }^{11}$ suggested that the local photovoltage created between metal nanoparticles and graphene causes the giant photoeffect. In their plausible mechanism, the generated photovoltage could funnel protons and electrons toward the metal nanoparticles for production of hydrogen, while repel holes away from them. However, this statement is not convincing on the basis of the static electric field theory, as protons and electrons have the 
opposite charge, and there is no further explanation about it. Otherwise, the microscopic mechanism analysis of this effect is missing in their work ${ }^{11}$.

Here we demonstrate a microscopic mechanism to explain the origin of the giant photoeffect in proton transport through the catalytically-activated graphene membrane. The local electric fields created between graphene and the metal nanoparticles induce dipole moments of electron orbits nearby. Upon photoexcitation, the local dipoles function as metastable electron states to trap hot electrons with relatively longer life time for effective reaction with injected protons to produce hydrogen. Herein, the catalyst increases the rate of a reaction by increasing the number of successful collisions of reactants instead of lowering the activation energy as usually expected.

\section{Results and discussion}

Let's begin our discussion from the origin of the steady proton current. The $100 \%$ Faradaic efficiency reported in the experiment ${ }^{11}$ implies that the steady current comes from the chemical reaction of electrons and protons on the other side of graphene. That is, there is no steady current without the chemical reaction. In the case of no chemical reaction, the bias voltage leads to a proton drift current from the proton source through graphene; while the different proton concentration causes an inverse proton diffusion current from the other side of graphene to the proton source. These two currents cancel each other out to reach equilibrium. So, there is no observed net steady current. The net proton diffusion current from the proton source through graphene produce when the chemical reaction occurs because chemical reaction reduces the proton concentration on the other side of graphene. The charge concentration on the other side of graphene should remain unchanged when a steady current is maintained, which gives

$$
\left|j_{\mathrm{p}}\right|=\left|j_{\mathrm{e}}\right|=q R_{\mathrm{ep}}
$$

where $j_{\mathrm{p}}$ and $j_{\mathrm{e}}$ are the current densities of protons and electrons respectively, $R_{\mathrm{ep}}$ is the reaction rate of electrons and protons per unit area and $q$ is the absolute charge of a proton or an electron. Because the chemical reaction has an energy barrier, only hot electrons with energy higher than the barrier energy can take part in the chemical reaction. The reaction rate is proportional to concentrations of protons and electrons that can take part in the chemical reaction: $R_{\mathrm{ep}}=r_{\mathrm{ep}} n p$, where $p$ and $n$ are concentrations of protons and hot electrons respectively, and $r_{\mathrm{ep}}$ is the intrinsic reaction coefficient. At equilibrium, the proton concentration can be tuned by the bias voltage, while the hot electrons concentration can be influenced by the incident photons. However, for the semi-metal pristine graphene, which has a continuous energy spectrum, most hot electrons excited by photons relax to lower energy states within a timescale of $10^{-12}$ $\mathrm{S}^{16}$, while the typical timescale of a chemical reaction is $10^{-6} \mathrm{~s}^{17,18}$. In other words, most hot electrons excited by the incident photons will relax to lower energy states before reacting with protons through graphene. This is the reason why no enhancement of the proton current under illumination was observed for graphene without decorating Pt nanoparticles in the experiment ${ }^{11}$.

In a macroscopic surface potential model, the surface electric field and free charge distribution are simplified to an abrupt model, i.e. the surface electric field is taken as a constant and the free charge is supposed to have a smooth even distribution, resulting a surface space charge region in a semiconductor ${ }^{19}$. However, many first principle calculations ${ }^{20-24}$ and theoretic models show that the free electrons in both the surface space charge region and the interface specific region (ISR) have modulated oscillatory density distributions rather than even distributions ${ }^{25}$. The interaction force between graphene and metal nanoparticles is electrostatic force. For graphene-metal equilibrium separation, electrons transfer from one to the other to equilibrate the Fermi levels because the work function of graphene differs from that of metal. This electron transfer results in the formation of interface dipole layer and an accompanying potential step. The local dipoles would be affected by the type and magnitude of the interaction force, because it is induced by the electric field of interfacial space charge layer. The lifetime of a hot electron is determined by the energy level of its ambient which is influenced by interfacial interaction forces. In the case of decorating Pt nanoparticles on graphene, the electrostatic potential at the contact area between Pt nanoparticle and graphene is different from that at the graphene surface without Pt nanoparticle. There are generally two classes of graphenemetal interfaces characterized by the binding energy between graphene and metal. Graphene forms strong interaction with the metal when the binding energy is greater than $2 \mathrm{eV}$, such as $\mathrm{Ni}$, $\mathrm{Pd}, \mathrm{Pt}, \mathrm{Co}, \mathrm{Ru}$. For the binding energy less than $2 \mathrm{eV}$, such as $\mathrm{Au}$, $\mathrm{Ag}, \mathrm{Cu}$, Ir, graphene forms weak interaction with these metals. When graphene contacts with metal by strong interaction ${ }^{26-31}$, the interfacial charge redistribution is not only the result of the electron transfer between the metal and the graphene levels. And the graphene-metal strong interaction plays a vital role in the formation of local dipoles as well ${ }^{21}$. For instance, the giant photoeffect was also observed for graphene decorated with other catalytically active metals $\mathrm{Pd}$ and $\mathrm{Ni}{ }^{32}$ known to strongly interact with graphene similar to Pt. In contrast, there is no such an effect when $\mathrm{Au}$ nanoparticles are used due to its weak interaction with graphene ${ }^{11}$. Hence, the graphene band structures are strongly perturbed and acquire a mixed graphenemetal character, leading to a modulated non-monotonic oscillatory potential in the ISR (Fig. 1) ${ }^{25,33}$. Herein, protons are trapped around the negative poles of the local dipoles while electrons are around the positive ones. Upon illumination, electrons are excited to metastable excited states with higher energy levels. Because of energy barriers around them, the free electrons at metastable excited states will have relatively longer lifetime which makes them possible to react with protons before relaxing to ground states. The local surface dipoles in the ISR serve as both proton collection centers and electron storage 


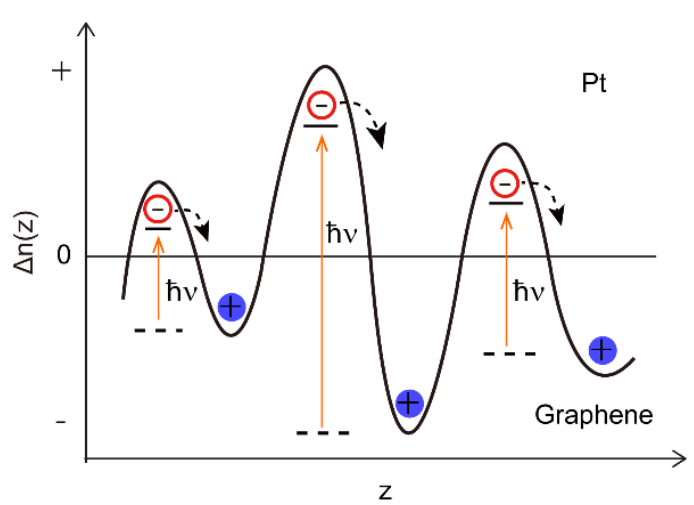

Fig. 1 Schematic illustration of the plane-averaged difference electron density $\Delta n$ along the $z$ direction showing the local dipoles formed in the specific region of the graphene-Pt nanoparticle interface. Protons are trapped around the negative poles of the local dipoles while negative free charges are near the positive ones. Electrons excited to metastable excited states with higher levels by photons can react with protons more effectively due to a relatively longer life time.

centers, and finally form chemical reaction centers. However, without illumination the electron concentration at the reaction center is low because of the high electric energy potential there. This leads to a small dark current as observed in the experiment ${ }^{11}$.

Under illumination the electrons at the ground state are transited to the metastable excited states with higher energy by the electric field of photons. Transitions between different metastable excited states may also happen. To simplify the discussion we employ an effective two level model to describe this phenomenon, with the framework showed in Fig. 2. The effective ground state is labeled as $|g\rangle$, and the effective excited state is labeled as $|e\rangle$. The energy difference between the excited state and the ground state is $E_{\mathrm{e}}$. The mean effective energy of photons is $\hbar \omega$, where $\omega$ is the mean effective angular frequency of photons. Generally there is an energy detuning between the energy difference of the two electron states and the photon energy: $\delta=E_{\mathrm{e}}-\hbar \omega$. The photon interacts with the electron via its electric field: $\widehat{H}_{\mathrm{I}}=-\vec{d} \cdot \vec{E}$, where $\vec{d}$ is the dipole moment of the electron and $\vec{E}$ is the electric field of the photon. The whole Hamiltonian can be simplified to the Jaynes-Cummings (JC) model by eliminating the high frequency part ${ }^{34}$ :

$$
\widehat{H}=\hbar \omega \hat{a}^{+} \hat{\mathrm{a}}+\left(E_{\mathrm{e}} / 2\right) \hat{\sigma}_{\mathrm{z}}+(h \Omega / 2)\left(\hat{a} \hat{\sigma}_{+}+\hat{a}^{+} \hat{\sigma}_{-}\right)
$$

where $\Omega$ is the photon-electron coupling constant, $\hat{a}^{+}$and $\hat{a}$ are creation and annihilation operators of photons respectively,

\begin{tabular}{lll} 
Excited state & \\
\cline { 2 - 3 } Energy detuning & & \\
Ground state & $\hbar \omega$ & $\vdots$ \\
& &
\end{tabular}

Fig. 2 Framework of the effective two level model. $\hat{\sigma}_{\mathrm{z}}=|e\rangle\langle e|-| g\rangle\left\langle g\left|, \hat{\sigma}_{+}=\right| e\right\rangle\langle g|$, and $\hat{\sigma}_{-}=|g\rangle\langle e|$. The Hamiltonian can be solved in a subspace $\{|m+1, g\rangle,|m, e\rangle\}$, where $m$ is the photon number density in graphene $\hat{a}^{+} \hat{a}|m\rangle=m|m\rangle$. The eigenstates and eigenvalues of the JC model are:

$$
\begin{aligned}
& |m,-\rangle=-\sin \left(\alpha_{m} / 2\right)|m, e\rangle+\cos \left(\alpha_{m} / 2\right)|m+1, g\rangle, \\
& E_{-}(m)=\hbar \omega(m+1 / 2)-\hbar \omega_{\mathrm{r}}(m, \delta) / 2 \\
& |m,+\rangle=\cos \left(\alpha_{m} / 2\right)|m, e\rangle+\sin \left(\alpha_{m} / 2\right)|m+1, g\rangle, \\
& E_{+}(m)=\hbar \omega(m+1 / 2)+\hbar \omega_{\mathrm{r}}(m, \delta) / 2,
\end{aligned}
$$

where $\omega_{\mathrm{r}}(m, \delta)=\sqrt{\delta^{2}+\Omega^{2}(m+1)}$ is the Rabi frequency and $\alpha_{m}=\tan ^{-1}(\Omega \sqrt{m+1} / \delta)$.

Because of the heating effect of illumination, the effective temperature of electrons under different illumination strength varies from each other and it is hard to predict the exact state of electrons without the knowledge of the exact temperature. Here we first resort to a very rough estimate of the excited electron state occupation by assuming a zero environment temperature and only the lowest eigenstate of the whole interacting Hamiltonian is occupied:

$$
|\langle e \mid m,-\rangle|^{2}=1 / 2\left(1-\delta / \omega_{\mathrm{r}}(m, \delta)\right)
$$

The concentration of high energy electrons is estimated as $n=\left(n_{0} / 2\right)\left(1-1 / \sqrt{I / I_{0}+1}\right)$, where $n_{0}$ is the effective total electron concentration, $I$ is the power density of the incident photon current and $I_{0}=I \delta^{2} /(m+1) \Omega^{2}=\hbar \omega \delta / \Omega^{2}$ is supposed to be a constant parameter. The equation shows that more electrons are pumped to the excited state under stronger illumination. The proton current density thus reads:

$$
j_{\mathrm{p}}=q R_{\mathrm{ep}}=q r_{\mathrm{ep}} p n=q r_{\mathrm{ep}} p\left(n_{0} / 2\right)\left(1-1 / \sqrt{I / I_{0}+1}\right) .
$$

For weak illumination $I<<I_{0}$ the proton current density has a linear dependence on the incident light power density:

$$
j_{\mathrm{p}} \approx q r_{\mathrm{ep}} p I\left(n_{0} / 4 I_{0}\right)
$$

while for strong illumination $I \gg I_{0}$ the proton current density saturates at:

$$
j_{\mathrm{p}} \approx q r_{\mathrm{ep}} p n_{0} / 2
$$

With finite environment temperature the concentration of high energy electrons is corrected as $n=\left(n_{0} / 2\right)\left(1-\tan h\left(\left(\hbar \delta / 2 k_{\mathrm{B}} T\right)\right.\right.$ $\left.\left.\sqrt{I / I_{0}+1}\right) \times 1 / \sqrt{I / I_{0}+1}\right)$, where $k_{\mathrm{B}}$ is the Boltzmann's constant, $T$ is the Kelvin temperature. The proton current density becomes:

$$
\begin{gathered}
j_{\mathrm{p}}=q r_{\mathrm{ep}}=q r_{\mathrm{ep}} p n=\left(q r_{\mathrm{ep}} p n_{0} / 2\right)\left(1-\tan h\left(\left(\hbar \delta / 2 k_{\mathrm{B}} T\right)\right.\right. \\
\left.\left.\sqrt{I / I_{0}+1}\right) \times 1 / \sqrt{I / I_{0}+1}\right)
\end{gathered}
$$

Let $a=q r_{\mathrm{ep}} p n_{0} / 2, b=I_{0}$ and $c=\hbar \delta / 2 k_{\mathrm{B}} T$ be three fitting parameters, the fitting curves are shown in Fig. 3, indicating a reasonable agreement with the experiment data of the proton current density ${ }^{11}$ although very rough approximations are made in the model. For weak illumination the heat effect is negligible and the temperature is invariant. While for strong illumination the proton current is underestimated by the model because of the heavy thermal effect. It should be noticed that the interface electric field distribution and the induced local dipole as described in the propose model could be impacted when metal particles have different sizes due to the quantum confinement effect. 


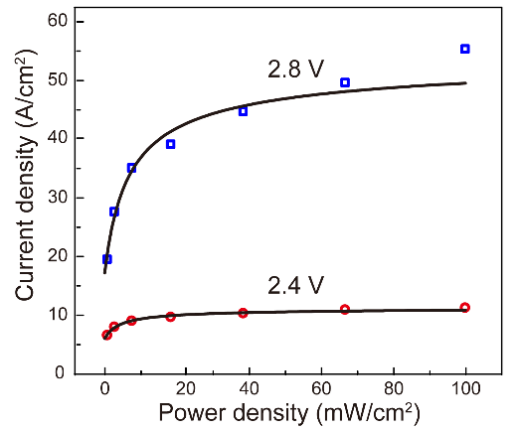

Fig. 3 Proton current density as a function of illumination power for different biases.

The effective two level model predicts proton current density curves (solid lines), which agree reasonably well with those of the experiment data (squares for $2.8 \mathrm{~V}$ and circles for $2.4 \mathrm{~V}$ of the bias, respectively) ${ }^{11}$.

Researchers have been attempted to take good advantage of hot carriers in photo(electro)catalysis, but the role is still not so significant ${ }^{35-39}$. On the basis of the above analysis, the giant photoeffect in proton transport through the catalyticallyactivated graphene membrane is attributed to long lived hot carries and fast proton transport rate. This reasoning is supported by many works. Since graphene is a strictly two-dimensional zero-bandgap semiconductor with a liner energy dispersion, it is theoretically predicted that the impact of carrier multiplication on photocurrent response might be enhanced by very inefficient electron cooling, resulting in an abundance of hot carriers ${ }^{40-42}$. Indeed, long lived hot carries have been reported in many experiments for graphene with built-in $\mathrm{p}-\mathrm{n}$ junctions ${ }^{43-45}$, explained as photovoltaic ${ }^{16,46-48}$ or photothermoelectric effect ${ }^{49,50}$. On the other hand, the rate for proton permeation through graphene is of the order of $10^{13}-10^{14} \mathrm{~s}^{-13}$, about 100 times faster than the lifetime of hot electrons, which has enough number to fully react with hot electrons for producing hydrogen. Taken together, the local dipoles induced by graphene and the metal nanoparticle function as metastable electron states with higher energy to trap hot electrons with relatively longer life time upon photoexcitation for effective reaction with large amount of injected protons to produce hydrogen. Since there is no change in the activation energy of the reaction, the metal catalyst increases the rate of a reaction by increasing the number of successful collisions of reactants for this significant photoeffect. This deduction is corroborated by the claim that the ratedetermining factor in the photocatalytic system is the concentration of surface-reaching photocharges before it reaches the threshold, instead of the widely accepted speculation of high reaction barriers to be overcome ${ }^{24,51}$. In most practical photo(electro)catalytic systems, the rapid photogenerated charge recombination ${ }^{52,53}$ and possible inhibit of mass transfer due to the complex electric double layer or ions in solution ${ }^{54-56}$ cause inferior performances.

\section{Conclusions}

In summary, we developed a microscopic mechanism about the new role of the catalyst based on the experiment of the illumination enhancement of proton transport through catalytically-activated graphene. Hot electrons in pristine graphene cannot participate a chemical reaction because of their short lifetime. When catalytically-active metal nanoparticles are decorated on graphene, they induce local dipoles in the interface specific region. Electrons can be trapped around the positive poles of the local dipoles, and protons are trapped around the negative ones after permeating through graphene. Upon illumination, electrons at the ground state can be pumped to metastable states with longer lifetime, so the chemical reaction between hot electrons and protons are accelerated. Our simplified two level model estimates the occupation of the excited electron state, and the corresponding fitting curves of the proton current density as a function of the incident light power density agree reasonably well with the experiment data. In the new mechanism the effect of metal nanoparticles is to stimulate local dipoles which provide long lived metastable excited states of electrons, instead of lowering the activation energy. A simplified effective two level model is also provided to explain the photocurrent density behavior under different illumination power density. This mechanism may shed light on the microscopic catalytic process and provide a guidance in designing photoelectrochemical energy conversion materials or devices. Furthermore, this mechanism can be generalized to other interfaces with the electrostatic interaction. Different from metal and semimetal systems, the lifetime of free electrons in semiconductor systems will be not extended by the formation of new metastable excited states because the conduction band edge of a semiconductor is actually a stable excited state. However, the function of local dipoles can be reflected in some microscopic and transient phenomenon such as photoluminescence spectra and the lifetime of photoluminescence.

\section{References}

(1) Achtyl, J. L.; Unocic, R. R.; Xu, L.; Cai, Y.; Raju, M.; Zhang, W.; Sacci, R. L.; Vlassiouk, I. V.; Fulvio, P. F.; Ganesh, P.; et al. Nat. Commun. 2015, 6, 6539. doi: 10.1038/ncomms7539

(2) Hu, S.; Lozada-Hidalgo, M.; Wang, F. C.; Mishchenko, A.; Schedin, F.; Nair, R. R.; Hill, E. W.; Boukhvalov, D. W.; Katsnelson, M. I.; Dryfe, R. A.; et al. Nature 2014, 516, 227. doi: 10.1038/nature14015

(3) Lozada-Hidalgo, M.; Hu, S.; Marshall, O.; Mishchenko, A.; Grigorenko, A. N.; Dryfe, R. A.; Radha, B.; Grigorieva, I. V.; Geim, A. K. Science 2016, 351, 68. doi: 10.1126/science.aac9726

(4) Lozada-Hidalgo, M.; Zhang, S.; Hu, S.; Esfandiar, A.; Grigorieva, I. V.; Geim, A. K. Nat. Commun. 2017, 8, 15215. doi: $10.1038 /$ ncomms 15215

(5) Kroes, J. M.; Fasolino, A.; Katsnelson, M. I. Phys. Chem. Chem. Phys. 2017, 19, 5813. doi: 10.1039/c6cp08923b

(6) Seel, M.; Pandey, R. 2D Materials 2016, 3, 025004. doi: $10.1088 / 2053-1583 / 3 / 2 / 025004$ 
(7) Shi, L.; Xu, A.; Chen, G.; Zhao, T. J. Phys. Chem. Lett. 2017, 8, 4354. doi: 10.1021/acs.jpclett.7b01999

(8) Bartolomei, M.; Hernández, M. I.; Campos-Martínez, J.; HernándezLamoneda, R. Carbon 2019, 144, 724. doi: 10.1016/j.carbon.2018.12.086

(9) Feng, Y.; Chen, J.; Fang, W.; Wang, E. G.; Michaelides, A.; Li, X. J. Phys. Chem. Lett. 2017, 8, 6009. doi: 10.1021/acs.jpclett.7b02820

(10) Poltavsky, I.; Zheng, L.; Mortazavi, M.; Tkatchenko, A. J. Chem. Phys. 2018, 148, 204707. doi: 10.1063/1.5024317

(11) Lozada-Hidalgo, M.; Zhang, S.; Hu, S.; Kravets, V. G.; Rodriguez, F. J.; Berdyugin, A.; Grigorenko, A.; Geim, A. K. Nat. Nanotechnol. 2018, 13, 300. doi: 10.1038/s41565-017-0051-5

(12) Linic, S.; Christopher, P.; Ingram, D. B. Nat. Mater. 2011, 10, 911. doi: $10.1038 /$ nmat 3151

(13) Brongersma, M. L.; Halas, N. J.; Nordlander, P. Nat. Nanotechnol. 2015, 10, 25. doi: 10.1038/nnano.2014.311

(14) Miao, M.; Nardelli, M. B.; Wang, Q.; Liu, Y. Phys. Chem. Chem. Phys. 2013, 15, 16132. doi: 10.1039/c3cp52318g

(15) Bunch, J. S.; Verbridge, S. S.; Alden, J. S.; van der Zande, A. M.; Parpia, J. M.; Craighead, H. G.; McEuen, P. L. Nano Lett. 2008, 8, 2458. doi: 10.1021/n1801457b

(16) Xia, F.; Mueller, T.; Lin, Y. M.; Valdes-Garcia, A.; Avouris, P. Nat. Nanotechnol. 2009, 4, 839. doi: 10.1038/nnano.2009.292

(17) Gimbert-Surinach, C.; Albero, J.; Stoll, T.; Fortage, J.; Collomb, M. N.; Deronzier, A.; Palomares, E.; Llobet, A. J. Am. Chem. Soc. 2014, 136, 7655. doi: 10.1021/ja501489h

(18) Hisatomi, T.; Takanabe, K.; Domen, K. Catal. Lett. 2014, 145, 95. doi: 10.1007/s10562-014-1397-z

(19) Kronik, L. Surf. Sci. Rep. 1999, 37, 1. doi: 10.1016/s0167-5729(99)00002-3

(20) Moglestue, C. J. Appl. Phys. 1986, 59, 3175. doi: 10.1063/1.336898.

(21) Gong, C.; Lee, G.; Shan, B.; Vogel, E. M.; Wallace, R. M.; Cho, K. J. Appl. Phys. 2010, 108, 123711. doi: 10.1063/1.3524232

(22) Zhu, H.; Zhou, C.; Wu, Y.; Lin, W.; Yang, W.; Cheng, Z.; Cai, X. Surf. Sci. 2017, 661, 1. doi: 10.1016/j.susc.2017.02.013

(23) Zhang, H. X.; Zhu, Y. F.; Zhao, M. Appl. Surf. Sci. 2017, 420, 105. doi: 10.1016/j.apsusc.2017.05.142

(24) Xie, G.; Guan, L.; Zhang, L.; Guo, B.; Batool, A.; Xin, Q.; Boddula, R.; Jan, S. U.; Gong, J. R. Nano Lett. 2019, 19, 1234. doi: 10.1021/acs.nanolett.8b04768

(25) Tung, R. T. Phys. Rev. B 2001, 64, 205310. doi: 10.1103/PhysRevB.64.205310

(26) Ran, Q.; Gao, M.; Guan, X.; Wang, Y.; Yu, Z. Appl. Phys. Lett. 2009, 94, 103511. doi: 10.1063/1.3095438

(27) Khomyakov, P. A.; Giovannetti, G.; Rusu, P. C.; Brocks, G.; van den Brink, J.; Kelly, P. J. Phys. Rev. B 2009, 79, 195425. doi: 10.1103/PhysRevB.79.195425

(28) Hupalo, M.; Liu, X.; Wang, C. Z.; Lu, W. C.; Yao, Y. X.; Ho, K. M.; Tringides, M. C. Adv. Mater. 2011, 23, 2082. doi: 10.1002/adma.201100412

(29) Gong, C.; Hinojos, D.; Wang, W.; Nijem, N.; Shan, B.; Wallace, R. M.; Cho, K.; Chabal, Y. J. ACS Nano 2012, 6, 5381. doi: $10.1021 / \mathrm{nn} 301241 \mathrm{p}$

(30) Pandey, P. A.; Bell, G. R.; Rourke, J. P.; Sanchez, A. M.; Elkin, M. D.; Hickey, B. J.; Wilson, N. R. Small 2011, 7, 3202. doi: $10.1002 / \mathrm{smll} .201101430$

(31) Lenz Baldez, R. N.; Piquini, P.; Schmidt, A. A.; Kuroda, M. A. Phys. Chem. Chem. Phys. 2017, 19, 22153. doi: 10.1039/c7cp04615d

(32) Mittendorfer, F.; Garhofer, A.; Redinger, J.; Klimeš, J.; Harl, J.; Kresse, G. Phys. Rev. B 2011, 84, 201401. doi: 10.1103/PhysRevB.84.201401

(33) Giovannetti, G.; Khomyakov, P. A.; Brocks, G.; Karpan, V. M.; van den Brink, J.; Kelly, P. J. Phys. Rev. Lett. 2008, 101, 026803. doi: 10.1103/PhysRevLett.101.026803

(34) Jaynes, E. T.; Cummings, F. W. Proc. IEEE 1963, 51, 89. doi: 10.1109 /proc. 1963.1664

(35) Sheldon, M. T.; van de Groep, J.; Brown, A. M.; Polman, A.; Atwater, H. A. Science 2014, 346, 828. doi: 10.1126/science.1258405

(36) Sobhani, A.; Knight, M. W.; Wang, Y.; Zheng, B.; King, N. S.; Brown, L. V.; Fang, Z.; Nordlander, P.; Halas, N. J. Nat. Commun. 2013, 4, 1643. doi: 10.1038/ncomms 2642

(37) Schuller, J. A.; Barnard, E. S.; Cai, W.; Jun, Y. C.; White, J. S.; Brongersma, M. L. Nat. Mater. 2010, 9, 193. doi: 10.1038/nmat2630

(38) Xu, Y. F.; Rao, H. S.; Chen, B. X.; Lin, Y.; Chen, H. Y.; Kuang, D. B.; Su, C. Y. Adv. Sci. 2015, 2, 1500049. doi: 10.1002/advs.201500049

(39) Wang, W.; Guo, B.; Dai, H.; Zhao, C.; Xie, G.; Ma, R.; Akram, M. Z.; Shan, H.; Cai, C.; Fang, Z.; et al. Nano Lett. 2019, 19, 6133. doi: 10.1021/acs.nanolett.9b02122

(40) Bistritzer, R.; MacDonald, A. H. Phys. Rev. Lett. 2009, 102, 206410. doi: 10.1103/PhysRevLett.102.206410

(41) Winzer, T.; Knorr, A.; Malic, E. Nano Lett. 2010, 10, 4839. doi: $10.1021 / \mathrm{nl} 1024485$

(42) Song, J. C.; Rudner, M. S.; Marcus, C. M.; Levitov, L. S. Nano Lett. 2011, 11, 4688. doi: 10.1021/n1202318u

(43) Gabor, N. M.; Song, J. C.; Ma, Q.; Nair, N. L.; Taychatanapat, T.; Watanabe, K.; Taniguchi, T.; Levitov, L. S.; Jarillo-Herrero, P. Science 2011, 334, 648. doi: 10.1126/science.1211384

(44) Tielrooij, K. J.; Piatkowski, L.; Massicotte, M.; Woessner, A.; Ma, Q.; Lee, Y.; Myhro, K. S.; Lau, C. N.; Jarillo-Herrero, P.; van Hulst, N. F.; et al. Nat. Nanotechnol. 2015, 10, 437. doi: 10.1038/nnano.2015.54

(45) Sun, D.; Aivazian, G.; Jones, A. M.; Ross, J. S.; Yao, W.; Cobden, D.; 
Xu, X. Nat. Nanotechnol. 2012, 7, 114. doi: 10.1038/nnano.2011.243

(46) Park, J.; Ahn, Y. H.; Ruiz-Vargas, C. Nano Lett. 2009, 9, 1742. doi: $10.1021 / \mathrm{n} 18029493$

(47) Mueller, T.; Xia, F.; Avouris, P. Nat. Photonics 2010, 4, 297. doi: $10.1038 /$ nphoton. 2010.40

(48) Nazin, G.; Zhang, Y.; Zhang, L.; Sutter, E.; Sutter, P. Nat. Phys. 2010, 6, 870. doi: $10.1038 /$ nphys 1745

(49) Xu, X.; Gabor, N. M.; Alden, J. S.; van der Zande, A. M.; McEuen, P. L. Nano Lett. 2010, 10, 562. doi: 10.1021/n1903451y

(50) Lemme, M. C.; Koppens, F. H.; Falk, A. L.; Rudner, M. S.; Park, H.; Levitov, L. S.; Marcus, C. M. Nano Lett. 2011, 11, 4134. doi: $10.1021 / \mathrm{nl} 2019068$

(51) Wang, D.; Sheng, T.; Chen, J.; Wang, H. F.; Hu, P. Nat. Catal. 2018,
1, 291. doi: 10.1038/s41929-018-0055-z

(52) Xie, G.; Zhang, K.; Guo, B.; Liu, Q.; Fang, L.; Gong, J. R. Adv. Mater. 2013, 25, 3820. doi: 10.1002/adma.201301207

(53) Walter, M. G.; Warren, E. L.; McKone, J. R.; Boettcher, S. W.; Mi, Q.; Santori, E. A.; Lewis, N. S. Chem. Rev. 2010, 110, 6446. doi: $10.1021 / \operatorname{cr} 1002326$

(54) Du, C.; Yang, X.; Mayer, M. T.; Hoyt, H.; Xie, J.; McMahon, G.; Bischoping, G.; Wang, D. Angew. Chem. Int. Ed. 2013, 52, 12692. doi: 10.1002/anie.201306263

(55) Waegele, M. M.; Gunathunge, C. M.; Li, J.; Li, X. J. Chem. Phys. 2019, 151, 160902. doi: 10.1063/1.5124878

(56) Ali, H.; Golnak, R.; Seidel, R.; Winter, B.; Xiao, J. ACS Appl. Nano Mater. 2019, 3, 264. doi: 10.1021/acsanm.9b01939 